\title{
Mathematical Model for Secondary Transport of Cations at the Root of Plants
}

\author{
Byunghyun Ban \\ bhban@kaist.ac.kr \\ Andong district office, Ministry of Employment and Labor.
}

\begin{abstract}
A mathematical expression to describe cation absorption of root is expressed with simulation results. The root cells selectively emit $\mathrm{H}+$ ions with active transport consuming ATPs to establish electrical gradient. The gradient promotes external positive ions to flow into the roots, while carries negatively charged particles with symport. In this paper, a mathematical model whose independent variables are the concentrations of external and internal cation is presented. This differential equation is induced from Ohm's law. The equation has terms for plant physiology, ion's physical and electrical properties, growth of plant, and interaction between the root and the surroundings. Simulation showed that the physiology-related coefficient has important role on nutrition absorption.
\end{abstract}

\section{Keywords}

aquaculture; computational biology; differential equation; ion transport; systems biology

\section{Introduction}

Recently, ODE network simulation on nutrient solution system to understand the black-boxed behavior of complex chemical system (Byunghyun Ban et al. 2019). The network based approach describes sedimentation and complex bidirected interactions among chemical compounds which the nutrient solution contains. It provided time-series simulations to investigate nutrient solution system with high accuracy. This method is based on ordinary differential equation (ODE) expression of chemical reactions. For example, ionization of $\mathrm{NaCl}_{(\mathrm{aq})}$ is described as equation (1-3), where $\mathrm{k}_{1}$ is forward reaction rate and $\mathrm{k}_{2}$ is backward reaction rate.

$$
\begin{aligned}
& \frac{d\left[\mathrm{Na}^{+}\right]}{d t}=k_{1}[\mathrm{NaCl}]-k_{2}\left[\mathrm{Na}^{+}\right]\left[\mathrm{Cl}^{-}\right] \\
& \frac{d\left[\mathrm{Cl}^{-}\right]}{d t}=k_{1}[\mathrm{NaCl}]-k_{2}\left[\mathrm{Na}^{+}\right]\left[\mathrm{Cl}^{-}\right] \\
& \frac{d[\mathrm{NaCl}]}{d t}=-k_{1}[\mathrm{NaCl}]+k_{2}\left[\mathrm{Na}^{+}\right]\left[\mathrm{Cl}^{-}\right]
\end{aligned}
$$

With reliable reaction rate coefficients, the equations can describe the concentration change of chemical components along time-scale. Combination of those reactions enables simulation of complex chemical system's behavior.

However, appropriate modeling method to match ODE scaled ion rate expression with plant's ion uptake is necessary to apply those complex-system simulation approach on prediction of plant's ion uptake. Although the influx of ions into roots are widely studied (Epstein and Hagan 1952; Epstein et al. 1963; Kochian and Lucas 
1982; Classen and Barber 1974; Nielsen 1976; Siddiqi and Glass 1982, 1986; Cram 1983; Buysse et al. 1996), their methods are not applicable to timescale simulation. First of all, they considered influx and efflux of ion as irreversible process so needed to prepare 2 separate models for each ion. Second, they did not distinguish flux of cation and anion. Cation influx is based on secondary transport, while plant absorbs anion with symport.

M. Silberbush and J.H. Lieth (2004) presented nitrate and potassium uptake prediction model but it is regression-based model to predict individual ion so does not consider the reaction of nitrate or potassium with other compounds to form plant-unabsorbable state.

An empirical model for macronutrient uptake is presented by A. Pardossi et al (2005) but they acquired data once a week so this model is not applicable to short-time interval simulation. Daniele Massa et al. (2008) presented an empirical model for sodium absorption simulation but this model ignores interference of other ionic components on the concentration of $\mathrm{Na}^{+}$; for example, reverse reaction of dissociation of $\mathrm{NaCL}$ or NaFeEDTA reduces the concentration of sodium ion.

So a continuous time scale available model which describes secondary transportation of cation is necessary.

\section{Materials and Methods}

I first considered the ion channels for cation absorption as electrical register and the other region of cell membrane as insulator. Then the group of a random ion channels becomes a parallel register system. Then the membrane potential $\mathrm{V}_{\mathrm{m}}$ induced by target ion gradient is the voltage, applied on the combined register $\mathrm{R}_{\text {ion }}$. Equation (4) is application of Ohm's law on this model, where $\mathrm{I}_{\text {ion }}$ is electric current of ion influx.

$$
\mathrm{I}_{\text {ion }}=\frac{1}{\mathrm{R}_{\mathrm{ion}}} V_{m}
$$

Electric current is described as equation (5). S is surface area where the charged particle passes, $\vec{v}$ is the average velocity of charged particle, $\mathrm{k}$ is the number of electrons which the selected ion lost, e is the charge of an electron and $n$ is the number of particle per unit volume. The equation has negative value because the flux of positively charged particle is opposite to electric current, which is induced by negatively charged electrons.

$$
\begin{gathered}
\mathrm{I}_{\mathrm{ion}}=-\mathrm{S} \bar{v} k e n \\
\mathrm{n}=-\frac{\mathrm{I}_{\mathrm{ion}}}{\text { Sivke }}
\end{gathered}
$$

As $\mathrm{n}$ is the number of particle in 1L volume, dividing both side of equation (6) Avogadro's number becomes equation of molarity. $N_{F}$ is Faraday's number, which is multiplication of $\mathrm{N}_{\mathrm{A}}$ and e. [ion] is the ion concentration inside the root because the electric current was defined as influx.

$$
\frac{\mathrm{n}}{\mathrm{N}_{\mathrm{A}}}=[\text { ion }]=-\frac{1}{N_{F}} \cdot \frac{\mathrm{I}_{\mathrm{ion}}}{S \bar{v} k}
$$

Differentiation on equation (7) expresses the differential equation of ion concentration change on time, which has same dimension with equation (1-3). 


$$
\frac{d[i o n]}{\mathrm{dt}}=-\frac{1}{N_{F}} \cdot \frac{1}{S \bar{v} k} \frac{d}{d t} I_{\text {ion }}
$$

Applying equation (4) on equation (8) leads to equation (10).

$$
\begin{gathered}
\frac{d}{d t} \mathrm{I}_{\text {ion }}=\frac{1}{\mathrm{R}_{\mathrm{ion}}} \frac{d}{d t} V_{m} \\
\frac{d[\text { ion }]}{\mathrm{dt}}=-\frac{1}{N_{F}} \cdot \frac{1}{S \bar{v} k} \frac{d}{d t} I_{\text {ion }}=-\frac{1}{N_{F}} \cdot \frac{1}{S \bar{v} k} \cdot \frac{1}{\mathrm{R}_{\mathrm{ion}}} \frac{d}{d t} V_{m}
\end{gathered}
$$

The force on a particle inside the electrical field is described as equation (11), where $\mathrm{E}$ is the electrical field, $\mathrm{q}$ is the charge of particle, $\theta$ is the thickness of membrane and $\mathrm{m}$ is the mass of ion. The acceleration of the particle is described as equation (12), where $m$ is the mass of single ion particle.

$$
\begin{array}{r}
\mathrm{F}=\mathrm{Eq}=\frac{\mathrm{V}_{m}}{\theta} \mathrm{q}=\mathrm{ma} \\
\mathrm{a}=\frac{\mathrm{dv}}{d t}=\frac{V_{m} k e}{m \theta}
\end{array}
$$

With the supposition that the force on cation during passing through the ion channel is uniform, and the initial velocity of ion is zero, the average velocity of the ion during transport is described as equation (13).

$$
\bar{v}=\sqrt{\frac{a \theta}{2}}=\sqrt{\frac{V_{m} k e \theta}{2 m \theta}}=\sqrt{\frac{V_{m} k e}{2 m}}
$$

Now applying equation (13) on equation (10) leads to equation (14).

$$
\frac{d[\text { ion }]}{\mathrm{dt}}=-\frac{1}{N_{F}} \cdot \frac{1}{S k} \cdot \sqrt{\frac{2 m}{V_{m} k e}} \cdot \frac{1}{\mathrm{R}_{\mathrm{ion}}} \frac{d}{d t} V_{m}
$$

Equation (15) describes the relationship between the resistance and permeability of ion channel. $P_{\text {ion }}$ is the permeability of the ion at channels defined in derivation of Goldman's equation $\left(\mathrm{P}_{\mathrm{ion}}=\frac{D_{A}}{\theta}\right), \mathrm{D}_{\mathrm{A}}$ is diffusion constant and $\theta$ is the thickness of the membrane. $\kappa$ is a coefficient.

$$
\mathrm{R}_{\text {ion }}=\frac{1}{\kappa} \frac{1}{P_{\text {ion }}}=\frac{1}{\kappa} \frac{\theta}{D_{A}}
$$

The mass of an ion is defined as equation (16), where $\mathrm{M}$ is molar weight. 


$$
\mathrm{m}=\frac{1}{1000} \frac{\mathrm{M}}{N_{A}}
$$

By applying equation $(15,16)$ on equation (14), equation (17) is derived.

$$
\begin{gathered}
\frac{d[\text { ion }]}{\mathrm{dt}}=-\frac{1}{N_{F}} \cdot \frac{1}{S k} \cdot \sqrt{\frac{2 m}{V_{m} k e}} \cdot \frac{1}{\mathrm{R}_{\mathrm{ion}}} \frac{d}{d t} V_{m} \\
=-\frac{1}{N_{F}} \cdot \frac{1}{S k} \cdot \sqrt{\frac{2 m}{V_{m} k e}} \cdot \kappa \mathrm{P}_{\mathrm{ion}} \frac{d}{d t} V_{m} \\
=-\frac{1}{N_{F}} \cdot \frac{1}{S k} \cdot \sqrt{\frac{2 m}{V_{m} k e}} \cdot \kappa \frac{D_{A}}{\theta} \frac{d}{d t} V_{m} \\
=-\frac{1}{N_{F}} \cdot \frac{1}{S k} \cdot \sqrt{\frac{2 M}{1000 V_{m} k e N_{A}}} \cdot \kappa \frac{D_{A}}{\theta} \frac{d}{d t} V_{m} \\
=-\frac{1}{10 \sqrt{5}} \cdot \frac{1}{S} \cdot \frac{1}{k N_{F}} \cdot \sqrt{\frac{M}{V_{m} k N_{F}}} \cdot \kappa \frac{D_{A}}{\theta} \frac{d}{d t} V_{m} \\
=-\frac{1}{10 \sqrt{5}} \cdot \frac{1}{S} \cdot \frac{1}{\left(k N_{F}\right)^{1.5}} \cdot \sqrt{\frac{M}{V_{m}}} \cdot \kappa \frac{D_{A}}{\theta} \frac{d}{d t} V_{m} \\
=-\frac{\kappa}{10 \sqrt{5} \theta} \cdot \frac{1}{S} \cdot \frac{D_{A} \sqrt{M}}{\left(k N_{F}\right)^{1.5}} \cdot \frac{1}{\sqrt{V_{m}}} \frac{d}{d t} V_{m}(17) \\
=-17
\end{gathered}
$$

I defined the constant $\mathcal{B}$ term as equation (18). Because phospholipid bilayers have typical thickness, I considered the thickness of membrane as a constant number.

$$
\frac{\kappa}{10 \sqrt{5} \theta}=\mathcal{B}
$$

Then the equation (17) is simplified into equation (19).

$$
\frac{d[\text { ion }]}{\mathrm{dt}}=-\frac{\mathcal{B}}{S} \cdot \frac{D_{A} \sqrt{M}}{\left(k N_{F}\right)^{1.5}} \cdot \frac{1}{\sqrt{V_{m}}} \frac{d}{d t} V_{m}
$$

$\mathrm{V}_{\mathrm{m}}$ is calculated with Goldman's equation, which has the concentration of ions inside and outside the root as the only independent variable. Therefore, this model describes the variance of ion concentration on time with 


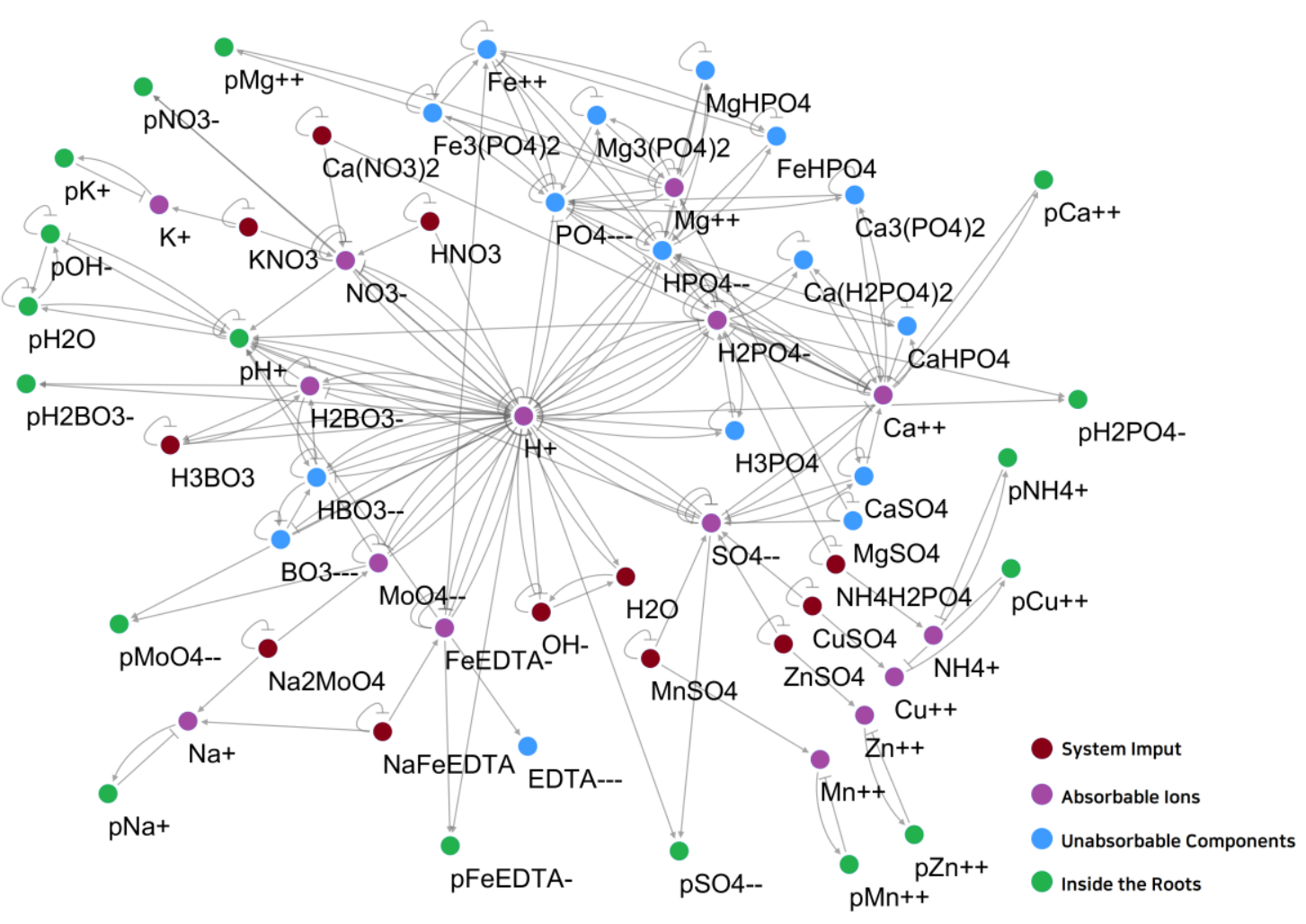

Figure 1. The network topology of plant and nutrient solution interaction model. Brown nodes are system input, inorganic fertilizers and water. Purple nodes are absorbable ions but blue nodes are unabsorbable. Green nodes indicate ions which are absorbed by the root, and water. The label of green nodes starts with small ' $p$ ' indicator.

respect to the ion concentration only.

The constant $\mathcal{B}$ describes the physiology of the plant species. It varies along the cell species because it is only affected by the membrane thickness, the diffusion constant of ion and combined resistance of ion channel. Membrane thickness of plant cells are not so different along the species and the diffusion constant is ion-related value. Therefore, $\mathcal{B}$ is only affected by the density and kind of ion channel.

$\mathrm{S}$ is the surface area where ion flows. It is defined as the inner area of ion channel multiplied with the number of ion channels. It is most influenced by the growth of plant's root. $D_{A} \sqrt{M}$ describes the physical property of the ion, while $\left(k N_{F}\right)^{1.5}$ describes the electrical property. $\frac{1}{\sqrt{V_{m}}} \frac{d}{d t} V_{m}$ can be derived with Goldman's equation. It depends on the environment, nutrient solution, and describes the interaction between the root cell and the surrounding.

Then I combined the ODE network simulation on nutrient solution system (Byunghyun Ban et al. 2019) with plant's ion absorption behavior. Detailed interactions, equations and coefficients are provided at https://github.com/needleworm, with the simulator and simulation results. The simulation has one supposition that the membrane gradient is mainly induced by $\mathrm{H}+$ ion only.

The topology of the complex system network model for interactions between nutrient solution and plant is described on the Figure 1. Figure 1 was generated with Cytoscape (Shannon P. et al. 2003). Total 20 simulations with different seeds were conduct. During simulations, reaction rate coefficients of chemical interactions and coefficient for $\mathrm{H}+$ ion pumping were fixed, while coefficients for plant-ion interactions were changed.

Also the initial state of systems was fixed. The nutrient solution state was Yamazaki's nutrient solution for lettuce (Yamazaki K., 1982). The plant was considered as an $1 \mathrm{~L}$ bag of H2O, containing no other nutrient inside. Coefficient sets for each experiment samples are presented on Supplementary Data 1. 
$\mathrm{pH}$ inside the root

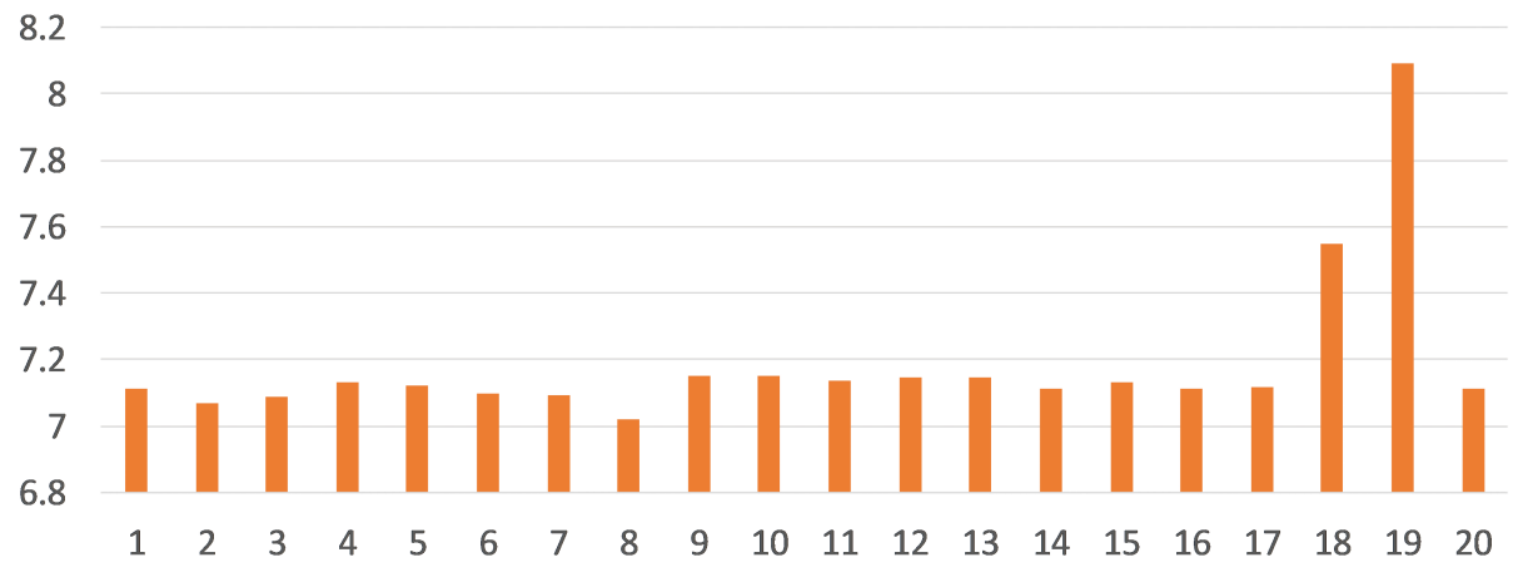

Figure $2 \mathrm{pH}$ inside the root at the convergence state.

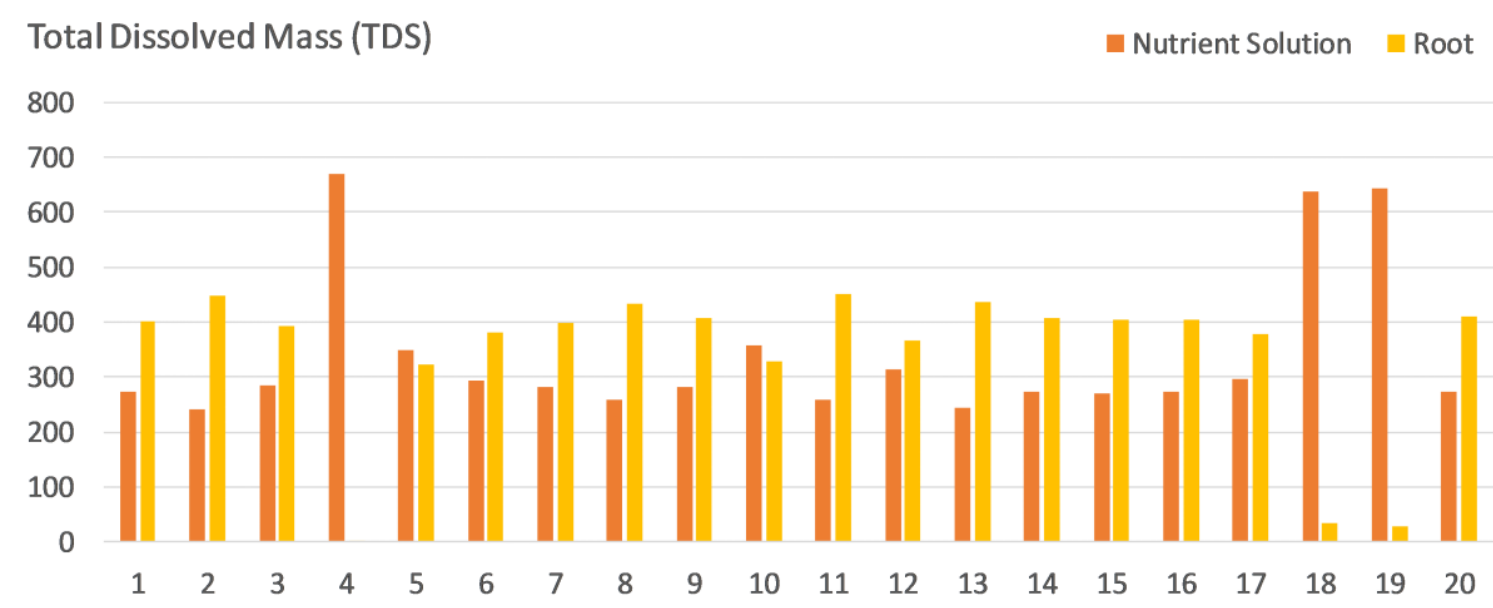

Figure 3 Total dissolved mass (TDS) of nutrient solution and the root. Root has higher TDS in most cases except trial 4, 5, 10, 18 and 19.

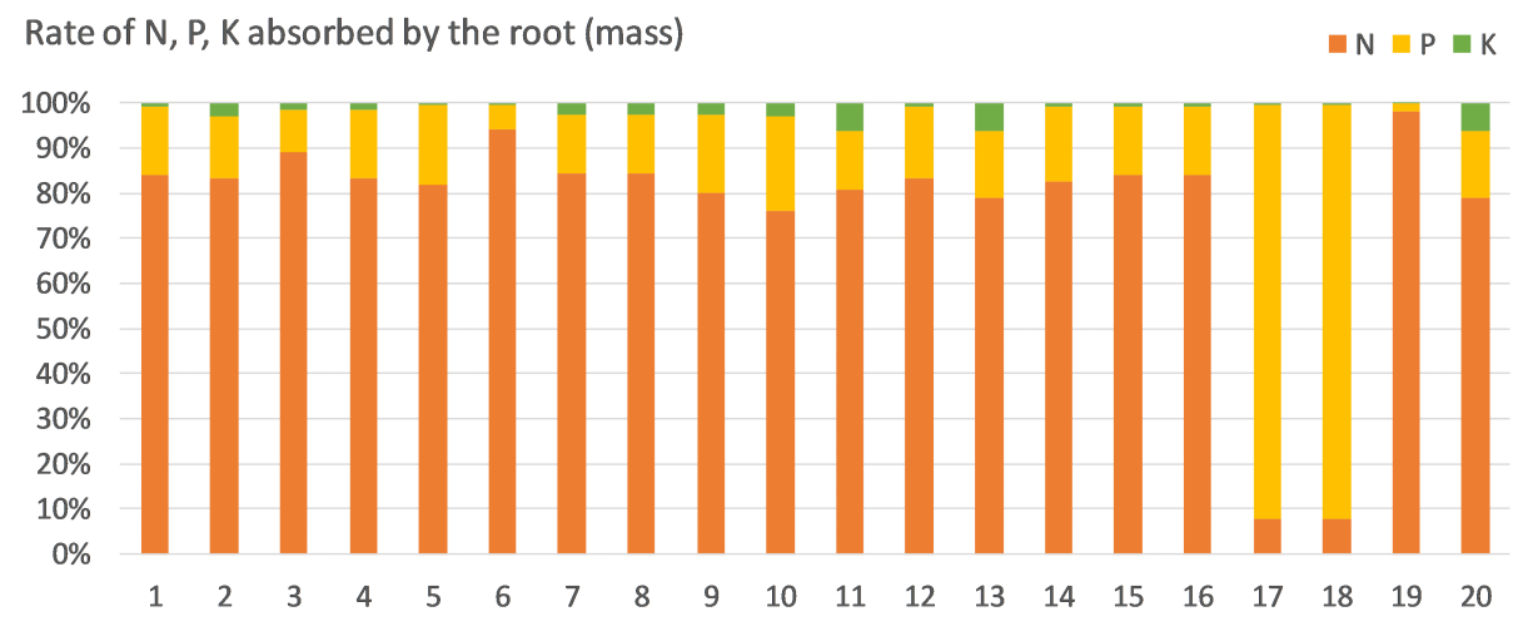

Figure 4 Mass rate of N, P, K inside the root, at the convergence state. 
The simulations were conducted until mean square error values between each gradient descent update reach 1e20. But trial 18 and 19 converged too fast so tighter MSE threshold was applied: 1e-21 and 1e-22 each.

\section{Results}

Experiment results are provided as Supplementary Data 2. Supplementary data 2 provides the system's convergence state.

The summaries of simulation experiments are presented on Figure 2-4. Figure 2 shows that $\mathrm{pH}$ inside the root after convergence varies along the trials. Figure 3 shows the TDS values of nutrient solution and plant for each trial. The ratio between them indicates the efficiency of nutrient absorption at the root. For example, trial 4, 18 and 19 showed the sample failed to absorb enough nutrient from the fertilizer. Figure 4 presents the rate of N, P and $\mathrm{K}$ absorbed by the root in mass scale. Trial 17 and 18 failed to absorb enough nitrogen and some samples seems to failed to absorb enough $\mathrm{K}$ ion.

\section{Discussion}

The result shows that different coefficient $\mathcal{B}$ values for same network topology can lead to different results. As $\mathcal{B}$ is a description of ion channel density on the cell membrane, it is considered as physiological term resulting from the gene expressions. Therefore each plant species has different $\mathcal{B}$ values, leading to different recipe of fertilization. For example, the nutrient solution recipes for lettuce and Basil are different because their ion consumption and absorption rate are different. Such phenomenon is understood as the result of different $\mathcal{B}$ coefficient.

The simulation also showed that plants with different $\mathcal{B}$ has different nutrition absorption rate, different $\mathrm{pH}$ and different TDS at the roots. Distinguishing $\mathcal{B}$ of unknown plant will help researchers to figure out nutrient solution recipes.

\section{Conclusion}

A differential equation model was induced from Ohm's law. The equation has $\mathcal{B}$ for plant physiology, $\mathrm{S}$ for the growth, $D_{A} \sqrt{M}$ for physical properties of ion, $\left(k N_{F}\right)^{1.5}$ for electrical properties of ion and $\frac{1}{\sqrt{V_{m}}} \frac{d}{d t} V_{m}$ for interaction between the environment and the plant. The simulation showed that different $\mathcal{B}$ values leads to different behaviors during nutrition absorption. Distinguishing $\mathcal{B}$ of various plant with experiments will enable high-precision prediction of ion consumption of crops. It will provide in-silico prediction of appropriate nutrient solution recipe for unknown species.

\section{Supplementary Data}

Supplementary Data 1 provides 20 different coefficient sets used for the experiments.

Supplementary Data 2 provides the convergence state values of each experiments.

\section{Acknowledgements}

Thanks to Minwoo Lee for giving me useful comments and delicious dinners.

\section{Data availability}

Experimental conditions and data are provided as supplementary data. The simulator and network models used 
for the experiment is freely available at https:/github.com/needleworm/nutrient_solution

\section{References}

Ban, B., Lee, M., \& Ryu, D. (2019). ODE network model for nonlinear and complex agricultural nutrient solution system. arXiv preprint arXiv:1907.10800.

Buysse, J., Smolders, E., Merckx, R., 1996. Modelling the uptake of nitrate by a growing plant with an adjustable root nitrate uptake capacity: I. Model description. Plant Soil 181, 19-23.

Claassen, N., Barber, S.A., 1974. A method for characterizing the relation between nutrient concentration and flux into roots of intact plants. Plant Physiol. 54, 564-568.

Cram, W.J., 1983. Chloride accumulation as a homeostatic system: set points and perturbations. The physiological significance of influx isotherms, temperature effects and the influence of plant growth substances. J. Exp. Bot. 34, 1484-1502.

Epstein, E., Hagen, C.E., 1952. A kinetic study of the absorption of alkali cations by barley roots. Plant Physiol. 27, 457-474.

Epstein, E., Rains, D.W., Elzam, O.E., 1963. Resolution of dual mechanisms of potassium absorption by barley roots. Proc. Natl. Acad. Sci. 49, 684-692.

Kochian, L.V., Lucas, W.J., 1982. Potassium transport in corn roots: I. Resolution of kinetics into a saturable and linear component. Plant Physiol. 70, 1723-1731.

Massa, D., Mattson, N. S., \& Lieth, H. (2008). An empirical model to simulate sodium absorption in roses growing in a hydroponic system. Scientia horticulturae, 118(3), 228-235.

Nielsen, N.E., 1976. A transport kinetic concept for ion uptake by plants: III. Test of the concept by results from water culture and pot experiments. Plant Soil 45, 659-677.

Pardossi, A., Falossi, F., Malorgio, F., Incrocci, L., \& Bellocchi, G. (2005). Empirical models of macronutrient uptake in melon plants grown in recirculating nutrient solution culture. Journal of plant nutrition, 27(7), 12611280 .

Shannon, P., Markiel, A., Ozier, O., Baliga, N. S., Wang, J. T., Ramage, D., ... \& Ideker, T. (2003). Cytoscape: a software environment for integrated models of biomolecular interaction networks. Genome research, 13(11), 2498-2504.

Siddiqi, M.Y., Glass, A.D.M., 1982. Simultaneous consideration of tissue and substrate potassium concentration in Kq uptake kinetics: a model. Plant Physiol. 69, 283-285.

Siddiqi, M.Y., Glass, A.D.M., 1986. A model for the regulation of Kq influx, and tissue potassium concentrations by negative feedback effects upon plasmalemma influx. Plant Physiol. 81, 1-7.

Silberbush, M., \& Lieth, J. H. (2004). Nitrate and potassium uptake by greenhouse roses (Rosa hybrida) along successive flower-cut cycles: a model and its calibration. Scientia horticulturae, 101(1-2), 127-141.

Yamazaki K. Nutrient solution culture. Tokyo, Japan: Pak-kyo Co; 1982. Japane 